Research Paper

\title{
Analysis of Clinical and Dosimetric Factors Influencing Radiation-Induced Lung Injury in Patients with Lung Cancer
}

\author{
Shuiyun Han 1,2, Feiying Gu 2 ${ }^{2}$ Gang Lin ${ }^{1,2}$, Xiaojiang Sun², Yuezhen Wang2, Zhun Wang2, Qingren Lin², \\ Denghu Weng ${ }^{2}$, Yaping $\mathrm{Xu}^{1,2 \varpi}$, Weimin $\mathrm{Mao}^{3}$ \\ 1. First Clinical Medical School, Wenzhou Medical University, Wenzhou, China \\ 2. Department of Radiation Oncology, Zhejiang Cancer Hospital, Hangzhou China \\ 3. Department of Thoracic Surgery, Zhejiang Cancer Hospital, Hangzhou, China \\ $\triangle$ Corresponding author: Yaping Xu, MD, 1.First Clinical Medical School, Wenzhou Medical University, Wenzhou, China, 2. Department of Radiation Oncol- \\ ogy, Zhejiang Cancer Hospital, Hangzhou China, 38 Guangji Road, Hangzhou, Zhejiang 310022, P.R. China; Telephone: +8613857101269; Fax: +86057188368079; \\ Email: xuyp@zjcc.org.cn
}

(c) 2015 Ivyspring International Publisher. Reproduction is permitted for personal, noncommercial use, provided that the article is in whole, unmodified, and properly cited. See http://ivyspring.com/terms for terms and conditions.

Received: 2015.04.02; Accepted: 2015.07.19; Published: 2015.09.15

\begin{abstract}
Purpose: Dose escalation of thoracic radiation can improve the local tumor control and surivival, and is in the meantime limited by the occurrence of radiation-induced lung injury (RILI). This study investigated the clinical and dosimetric factors influencing RILI in lung-cancer patients receiving chemoradiotherapy for better radiation planning.

Methods and Materials: A retrospective analysis was carried out on 161 patients with non-small-cell or small-cell lung cancer (NSCLC and SCLC, respectively), who underwent chemoradiotherapy between April 2010 and May 2011 with a median follow-up time of 545 days (range: 39-1453). Chemotherapy regimens were based on the histological type (squamous cell carcinoma, adenocarcinoma, or SCLC), and radiotherapy was delivered in 1.8-3.0 Gy (median, 2.0 Gy) fractions, once daily, to a total of 39-66 Gy (median, $60 \mathrm{~Gy}$ ). Univariate analysis was performed to analyze clinical and dosimetric factors associated with RILI. Multivariate analysis using logistic regression identified independent risk factors correlated to RILI.

Results: The incidence of symptomatic RILI ( $\geq$ grade 2 ) was $31.7 \%$. Univariate analysis showed that $\mathrm{V} 5, \mathrm{~V} 20$, and mean lung dose (MLD) were significantly associated with RILI incidence $(P=0.029$, 0.048 , and 0.041 , respectively). The association was not statistically significant for histological type (NSCLC vs. SCLC, $P=0.092$ ) or radiation technology (IMRT vs. 3D-CRT, $P=0.095$ ). Multivariate analysis identified MLD as an independent risk factor for symptomatic RILI (OR=1.249, $95 \% \mathrm{Cl}=1.055-1.48, P=0.01$ ). The incidence of bilateral $\mathrm{RILI}$ in cases where the tumor was located unilaterally was $22.7 \%(32 / 141)$ and all dosimetric-parameter values were not significantly different $(P>0.05)$ for bilateral versus ipsilateral injury, except grade-1 (low) RILI $(P<0.05)$. The RILI grade was higher in cases of ipsilateral lung injury than in bilateral cases (Mann-Whitney $U$ test, $z=8.216$, $P<0.001$ ).

Conclusion: The dosimetric parameter, MLD, was found to be an independent predictive factor for RILI. Additional contralateral injury does not seem to be correlated with increased RILI grade under the condition of conventional radiotherapy treatment planning.
\end{abstract}

Key words: lung cancer; radiation-induced lung injury; Dose-volume-histogram

\section{Introduction}

Radiation therapy forms part of the backbone of lung-cancer treatment, and research has shown that, with each additional Gy of radiation, long-term (3-5 year) locoregional tumor control is absolutely im- 
proved by $1 \%$, and that the risk of death is reduced by $3 \%$ for non-small-cell lung cancer (NSCLC) ${ }^{1}$. Patients with small cell lung cancer (SCLC) can obtain an absolute survival increase of $5 \%$ at 2 and 3 years respectively with the addition of thoracic radiotherapy ${ }^{2,3}$. Despite the improved capacity of modern radiation techniques to increase survival rate and lessen radiation-related toxicity via more localized dose delivery, radiation induced lung injury (RILI) remains a dose-limiting complication that hinders treatment and worsens patient quality of life. Clinical presentation is similar to pneumonitis but it is usually nonbacterial in the acute phase (named radiation pneumonitis, RP).

Clinical factors including gender ${ }^{4}$, tumor location 5 , age 4,6 , and administration of induction gemcitabine ${ }^{6}$ have been shown to be associated with the development of RILI, but some studies have reported the opposite 7,8. Many studies have identified parameters relating to the dose-volume histogram (DVH) that are associated with the occurrence of RILI; these include $\mathrm{V}_{5}{ }^{9}$, V20 7,10-13, V30 8,12,14,15, and mean lung dose (MLD) $4,7,12,15$. The percentage split of the Vdose (defined as the percentage of lung volume irradiated with doses exceeding a threshold) and the MLD (e.g.17.7-Gy split by Zhang et al 7, 17.4-Gy split by Wang et al ${ }^{4}$ ) differs across studies.

The above inconsistencies in the literature make clinical adoption of RILI-related reference parameters difficult. In this study, we investigated clinical and dosimetric risk factors for RILI in 161 patients with NSCLC or SCLC, who received concurrent or sequential chemoradiotherapy in our department.

\section{Methods and Materials}

\section{Study population}

This retrospective review of medical records was approved by the ethics committee of Zhejiang Cancer Hospital, Hangzhou, China, and signed informed consents were obtained from all patients before starting therapy. Eligible patients were included according to the following criteria: (1) histologically or cytologically confirmed NSCLC or SCLC, (2) follow-up time of at least 3 months or observed endpoint, (3) consecutive patients received radiotherapy April 2010 to May 2011, (4) no basic cardiopulmonary diseases, (5) no previous thoracic radiation therapy and (6) no contralateral pleural nodules or separate tumor nodule (s) in the contralateral lobe.

\section{Treatment}

Regimens of conventionally fractionated radiotherapy combined with chemotherapy (synchronous or sequential) were delivered to all patients; each received either intensity-modulated radiotherapy (IMRT) or three-dimensional conformal radiotherapy
(3D-CRT). All the patients underwent simulation CT scans with enhancement. The gross tumor volume (GTV) consisted of the primary tumor and the affected lymph nodes or hilum zones. The clinical target volume (CTV) was obtained by $6-8-\mathrm{mm} 3 \mathrm{D}$ expansion on the basis of the GTV, and the planning target volume (PTV) was generated by additional expansion not exceeding $1.5 \mathrm{~cm}$, considering the microscopic tumor extension, mobility, and daily setup errors. Physicians may necessarily reference PET/CT during delineation.

Radiation treatment plans were calculated with Pinnacle treatment planning system software (Philips Medical Systems), according to the criterion that at least $95 \%$ of the PTV was delivered the prescribed dose. Tissue inhomogeneity corrections were applied for all plans.

Patients with NSCLC received cisplatin doublets (with gemcitabine, paclitaxel, etoposide, pemetrexed, or vinorelbine) and etoposide/platinum was used for more than $90 \%$ of small-cell lung cancer.

\section{Diagnosis and follow-up}

The diagnosis of RILI was made based on radiographic abnormalities combined with clinical symptoms and signs. We scored RILI as follows, using CTCAE4.0 as a reference. grade 1: asymptomatic, clinical or diagnostic observations only, intervention not indicated; grade 2: symptomatic, medical intervention indicated, impairment of normal daily activities; grade 3: severe or medically significant but not immediately life-threatening, hospitalization or prolongation of hospitalization indicated, self-care activities impaired; grade 4: life-threatening respiratory consequences, urgent intervention indicated (e.g., tracheotomy or intubation); grade 5: death due to RILI. A grade of 2 or above was defined as symptomatic RILI and this was the main observation endpoint.

Patients underwent CT scans one month after completion of radiotherapy, with follow-ups every 3 months for 2 years, then every 6 months for the next 3 years, and every 2 years thereafter. Evaluation at the time of follow-up included a history, physical examination, and CT scans. Brain CT scans and bone scans were obtained every 1 year, unless the clinical situation dictated an increase in the frequency.

\section{Statistical analysis}

Statistical analysis was carried out using IBM SPSS 21.0 according to the distribution of data. Percentages were compared between groups using the chi-squared test, and normally distributed dosimetric factors were compared using a t-test. Logistic regression was performed for multivariate analysis of 
symptomatic RIL using a backward regression procedure including the clinical and dosimetric factors with a $P$-value $<0.1$ in the univariate analysis. The association between variables was tested using Pearson's or Spearman's correlation coefficients. A Mann-Whitney $U$ test was carried out to examine the distribution of RILI grades between groups. A $P$-value of 0.05 or less was considered statistically significant (two-tailed).

\section{Results}

\section{Patient characteristics}

A total of 161 consecutive patients received thoracic radiotherapy between April 2010 and May 2011. Table 1 shows the general characteristics of this set of patients. Ages ranged from 23-65 years and the median was 56. NSCLC accounted for $65.2 \%$ of the cases $(n=105)$, consisting of 55 squamous cell carcinomas, 46 adenocarcinomas, 3 no specific type and 1 adenosquamous carcinoma. Of the patients with stage-IV diagnosis, there were 5 cases of bone metastasis, 5 of brain metastasis, 2 of ipsilateral localized pleural metastasis, 2 of adrenal metastasis, and 1 of submandibular lymph node metastasis. The median total radiation dose delivered was $60 \mathrm{~Gy}$ ) at 2 Gy/fraction (range: 39-66 Gy at 1.8-3 Gy/fraction), and median radiation duration was 42 days (range: 22-61 days). The range of follow-up times was 39-1453 (median: 545 days).

Table 1. Patient characteristics

\begin{tabular}{lll}
\hline Factors & & Value \\
\hline Gender & Male & 137 \\
Age (years) & Female & 24 \\
Smoke & Median (range) & $56(23-76)$ \\
& $\geq 30$ pack-years & 109 \\
KPS & $<$ 30pack-years & 52 \\
& $\geq 90$ & 144 \\
Location & $<90$ & 17 \\
& Upper-lobe & 76 \\
Histological type & Other lobes & 85 \\
& Non small cell lung cancer & 105 \\
Stage & Small cell lung cancer & 56 \\
& I & 5 \\
& II & 23 \\
Surgery & III & 118 \\
& IV & 15 \\
Concomitant CRT & Yes & 42 \\
& No & 119 \\
Chemotherapy & No & 73 \\
& With gemcitabine & 88 \\
Chemothrapy cycles & Without gemcitabine & 38 \\
Technology & Median (range) & 123 \\
& 3D-CRT & $4(1-8)$ \\
Radiation dose & IMRT & 85 \\
\hline & Median (range),Gy & 76 \\
& & $60(39-66)$ \\
\hline
\end{tabular}

RILI: radiation induced lung injury; NSCLC: non-small cell lung cancer; SCLC: small cell lung cancer; CRT: chemoradiotherapy; KPS: Karnofsky performance status; 3D-CRT: 3D-corformal radiation therapy.
All patients were treated with combined radiation and chemotherapy regimens, but $42(26.1 \%)$ also underwent surgery. Of these, 41 received postoperative radiotherapy and 1 received neoadjuvant chemoradiotherapy. Of the total patient sample with a median chemotherapy cycle was 4 cycles (range: 1-8 cycles), $45.3 \%$ were treated via concurrent chemoradiation without gemcitabine. Of 105 patients with NSCLC, 11 patients using gemcitabine regimen in induction chemotherapy were delivered another chemotherapy regimens during concurrent chemoradiation. Totally 38 patients were given with gemcitabine/platinum, 15 vinorelbine/cisplatin, 4 etoposide/platinum, 42 pemetrexed/platinum and 17 paclitaxel / platinum for chemotherapy. More than 90 percent of patients with SCLC received etoposide/platinum treatments. No chemotherapy-related death was recorded.

\section{The incidence of RILI}

The majority of patients did not develop symptomatic RILI, with $12.6 \%(\mathrm{n}=20)$ being RILI-free and $55.9 \%(\mathrm{n}=90)$ exhibiting only grade-1 RILI. The remaining $31.7 \%$ (51/161) were symptomatic (grade 2 : $n=35,21.7 \%$; grade $3: n=15,9.3 \%$; grade $5: n=1,0.6 \%$ ). The median time to RILI after the beginning of radiation treatment was 138 days (range: 9-1327), 7 patients developed radiation-related pneumonia during radiotherapy, and one of them stopped the radiation due to a grade-3 injury. One patient died of RILI.

We observed that in some cases where the tumor was located on one side of the lung only, RILI occurred bilaterally. Of the 141 patients who developed RILI, 32 (22.7\%) showed bilateral lung injury; 20\% (18/90), $25.7 \%(9 / 35)$, and $26.67 \%(4 / 15)$ of grade 1,2 and 3 cases, respectively.

\section{Factors affecting RILI}

The clinical and dosimetric factors associated with RILI are shown in Table 2. No clinical factors were significantly associated with RILI, but it tended to be lower in cases of small-cell lung cancer (NSCLC vs. SCLC: $36.2 \%$ vs. $23.2 \%$, chi-square value $2.841, P=$ 0.092) and where 3D-CRT had been used (3D-CRT vs. IMRT: $25.9 \%$ vs. $38.2 \%$, chi-square value $2.794, P=$ 0.095), but these effects were not statistically significant (univariate analysis). The percentage of pulmonary volume irradiated at 5 Gy (range: 13.56-49.84 \%) and 20 Gy (range: 5.97-28.54\%) was significantly higher in patients with symptomatic RILI versus non-symptomatic RILI ( $P=0.029$ and 0.048 , respectively). In contrast, V30 (range: 5.21-22.05\%) values were similar $(17.08 \pm 3.01 \%$ vs. $16.18 \pm 4.07 \%, \mathrm{t}=1.574, P=$ 0.118). Another significant dosimetric factor was MLD 
(range: $4.26-14.94 \mathrm{~Gy}, 12.9 \pm 1.88$ Gy vs. $12.11 \pm 2.41 \mathrm{~Gy}$, $\mathrm{t}=2.057, P=0.041)$.

The continuous distribution showed high correlation between V5, V20, and MLD with $\mathrm{r}$ values from 0.744 to 0.861 using Pearson correlation analysis; for this reason we chose MLD for the multivariate analysis. For this analysis, logistic regression was used to examine the influence of the histological type, the radiation technology used, and the MLD. Only MLD was identified as an independent risk factor for symptomatic RILI (OR=1.249, 95\% CI=1.055-1.48, $P=$ $0.01)$.

Table 2. Univariate analysis for clinical and dosimetric risk factors of RILI in 161 patients with lung cancer.

\begin{tabular}{|c|c|c|c|}
\hline Factor & $\geq$ Grade-2 RILI & < Grade-2 RILI & $P$ value \\
\hline \multicolumn{4}{|l|}{ Gender } \\
\hline Male & 43 & 94 & 0.85 \\
\hline Female & 8 & 16 & \\
\hline \multicolumn{4}{|l|}{ Age (years) } \\
\hline$\geq 65$ & 12 & 15 & 0.118 \\
\hline$<65$ & 39 & 95 & \\
\hline \multicolumn{4}{|l|}{ Smoke } \\
\hline$\geq 30$ pack-years & 31 & 78 & 0.201 \\
\hline$<30$ pack-years & 20 & 32 & \\
\hline \multicolumn{4}{|l|}{ KPS } \\
\hline$\geq 90$ & 47 & 97 & 0.445 \\
\hline$<90$ & 4 & 13 & \\
\hline \multicolumn{4}{|l|}{ Location } \\
\hline Upper-lobe & 24 & 52 & 0.98 \\
\hline Other lobes & 27 & 58 & \\
\hline \multicolumn{4}{|l|}{ Histologic type } \\
\hline NSCLC & 38 & 67 & 0.092 \\
\hline SCLC & 13 & 43 & \\
\hline \multicolumn{4}{|l|}{ Stage } \\
\hline $\mathrm{I}+\mathrm{II}$ & 8 & 20 & 0.698 \\
\hline III+IV & 43 & 90 & \\
\hline \multicolumn{4}{|l|}{ Surgery } \\
\hline Yes & 16 & 26 & 0.298 \\
\hline No & 35 & 84 & \\
\hline \multicolumn{4}{|l|}{ Concomitant CRT } \\
\hline Yes & 27 & 46 & 0.187 \\
\hline No & 24 & 64 & \\
\hline \multicolumn{4}{|l|}{ Chemotherapy } \\
\hline With gemcitabine & 14 & 24 & 0.434 \\
\hline Without gemcitabine & 37 & 86 & \\
\hline \multicolumn{4}{|l|}{ Chemotherapy cycles } \\
\hline$\geq 4$ & 33 & 75 & 0.662 \\
\hline$<4$ & 18 & 35 & \\
\hline \multicolumn{4}{|l|}{ Technology } \\
\hline 3D-CTR & 22 & 63 & 0.095 \\
\hline IMRT & 29 & 47 & \\
\hline \multicolumn{4}{|l|}{ Radiation dose (Gy) } \\
\hline$\geq 60$ & 31 & 59 & 0.395 \\
\hline$<60$ & 20 & 51 & \\
\hline Whole lung V5, \% & $41.37 \pm 6.04$ & $38.69 \pm 7.7$ & 0.029 \\
\hline Whole lung V20, \% & $23.8 \pm 3.23$ & $22.55 \pm 4.53$ & 0.048 \\
\hline Whole lung V30, \% & $17.08 \pm 3.01$ & $16.18 \pm 4.07$ & 0.118 \\
\hline Whole lung MLD, Gy & $12.9 \pm 1.88$ & $12.11 \pm 2.41$ & 0.041 \\
\hline
\end{tabular}

Table 3. Multivariate analysis of clinical and dosimetric risk factors for RILI in 161 patients with lung cancer.

\begin{tabular}{llllllll}
\hline & & & & & \multicolumn{3}{c}{ EXP (B) 95\% C.I. } \\
\hline & B & S.E, & Wals & Sig. & Exp (B) & Low & Upper \\
MLD & 0.223 & 0.086 & 6.634 & 0.01 & 1.249 & 1.055 & 1.48 \\
Technology & 0.504 & 0.366 & 1.894 & 0.169 & 1.656 & 0.807 & 3.395 \\
Histologic & 0.722 & 0.461 & 2.446 & 0.118 & 2.058 & 0.833 & 5.084 \\
type & & & & & & & \\
\hline
\end{tabular}

MLD: mean lung dose; RILI: radiation-induced lung injury

Table 4. Mean and $95 \% \mathrm{Cl}$ of $\mathrm{V} 5, \mathrm{~V} 20, \mathrm{~V} 30$, and MLD for each grade of RILI; cases of ipsilateral and bilateral injury are compared.

\begin{tabular}{|c|c|c|c|c|c|c|}
\hline & & Bilateral & & Ipsilateral & & \\
\hline & Group & Mean $\pm S D$ & $95 \% \mathrm{CI}$ & Mean \pm SD & $95 \% \mathrm{CI}$ & $P$ value \\
\hline V5 & All RILI & $42.84 \pm 5.41$ & $40.89-44.79$ & $39.15 \pm 7.06$ & $37.81-40.50$ & 0.007 \\
\hline V20 & & $24.62 \pm 3.18$ & $23.48-25.77$ & $22.91 \pm 4.05$ & $22.14-23.68$ & 0.03 \\
\hline V30 & & $17.91 \pm 3.02$ & $16.82-19.00$ & $16.39 \pm 3.67$ & $15.69-17.09$ & 0.034 \\
\hline MLD & & $13.30 \pm 2.10$ & $12.54-14.06$ & $12.28 \pm 2.15$ & $11.87-12.69$ & 0.019 \\
\hline V5 & RILI 1 & $43.68 \pm 4.86$ & $41.26-46.09$ & $38.09 \pm 7.31$ & $36.37-39.81$ & 0.003 \\
\hline V20 & & $25.38 \pm 2.67$ & $24.05-26.71$ & $22.43 \pm 4.4$ & $21.39-23.46$ & 0.008 \\
\hline V30 & & $18.73 \pm 2.56$ & $17.46-20.00$ & $15.99 \pm 3.97$ & $15.05-16.91$ & 0.001 \\
\hline MLD & & $13.85 \pm 2.10$ & $12.81-14.90$ & $11.98 \pm 2.24$ & $11.38-12.42$ & 0.002 \\
\hline V5 & RILI 2 & $42.35 \pm 4.26$ & $39.07-45.62$ & $41.74 \pm 6.14$ & $39.26-44.22$ & 0.785 \\
\hline V20 & & $23.72 \pm 2.69$ & $21.65-25.78$ & $24.04 \pm 3.16$ & $22.76-25.32$ & 0.787 \\
\hline V30 & & $17.26 \pm 2.5$ & $15.34-19.18$ & $16.83 \pm 3.04$ & $15.60-18.06$ & 0.705 \\
\hline MLD & & $12.59 \pm 1.45$ & $11.47-13.70$ & $12.98 \pm 2.04$ & $12.16-13.81$ & 0.600 \\
\hline V5 & RILI 3 & $38.74 \pm 9.05$ & 24.33-53.15 & $40.03 \pm 6.18$ & $35.88-44.18$ & 0.756 \\
\hline V20 & & $22.60 \pm 5.60$ & $13.69-31.50$ & $23.41 \pm 3.15$ & $21.30-25.53$ & 0.722 \\
\hline V30 & & $16.01 \pm 5.48$ & $7.30-24.73$ & $17.98 \pm 2.56$ & $16.26-19.70$ & 0.348 \\
\hline MLD & & $12.29 \pm 3.2$ & $7.20-17.38$ & $13.08 \pm 1.49$ & $12.07-14.08$ & 0.515 \\
\hline
\end{tabular}

\section{Discussion}

In this study, we have found a little higher incidence of symptomatic RILI $(31.7 \%)$ than has been reported in previous studies $(16.6 \%-30.6 \%)$ 10,11,15-17. One of the patients with RILI $\geq 2$ grade stopped the 
radiation treatment on account of its adverse effects, and another died because of radiation-induced lung toxicity. With the unavailability of specific drugs, treatment of RILI mainly consists of administering oxygen and steroids, and RILI remains a challenge for effective and safe radiation treatment. Our statistical work showed that dosimetric parameter like MLD was an important factor influencing the onset of symptomatic RILI (OR=1.249, 95\%CI=1.055-1.48, $P=$ $0.01)$. Thirty-two $(22.7 \%)$ patients was recorded bilateral lung injury, which seems not correlate with a high risk of higher-grade RILI under the acceptable dosimetric parameter from DVH.

Several clinical factors for radiation-induced lung injury have been reported, with sometimes discordant results; these include age ${ }^{4}$, gender ${ }^{18}$, tumor location ${ }^{5}$, gemcitabine ${ }^{13}$, and COPD ${ }^{17}$. In the present study, no statistically significant association was found between the clinical factors mentioned above and RILI, but a trend toward an association with histologic type (NSCLC vs. SCLC: $P=0.092$ ) and radiation technology (IMRT vs. 3D-CRT: $38.2 \%$ vs. $25.9 \%$, $P=0.095)$ was observed. The constraints on tissue dose-volume and the radiation plan are similar for NSCLC and SCLC; for this reason, we applied both SCLC and NSCLC to evaluating the impact of histological type on RILI and found that a higher percentage of SCLCs compared to other studies 7,8,11,16-18. However, multivariate analysis did not show that histological type was an independent predictive value of RILI.

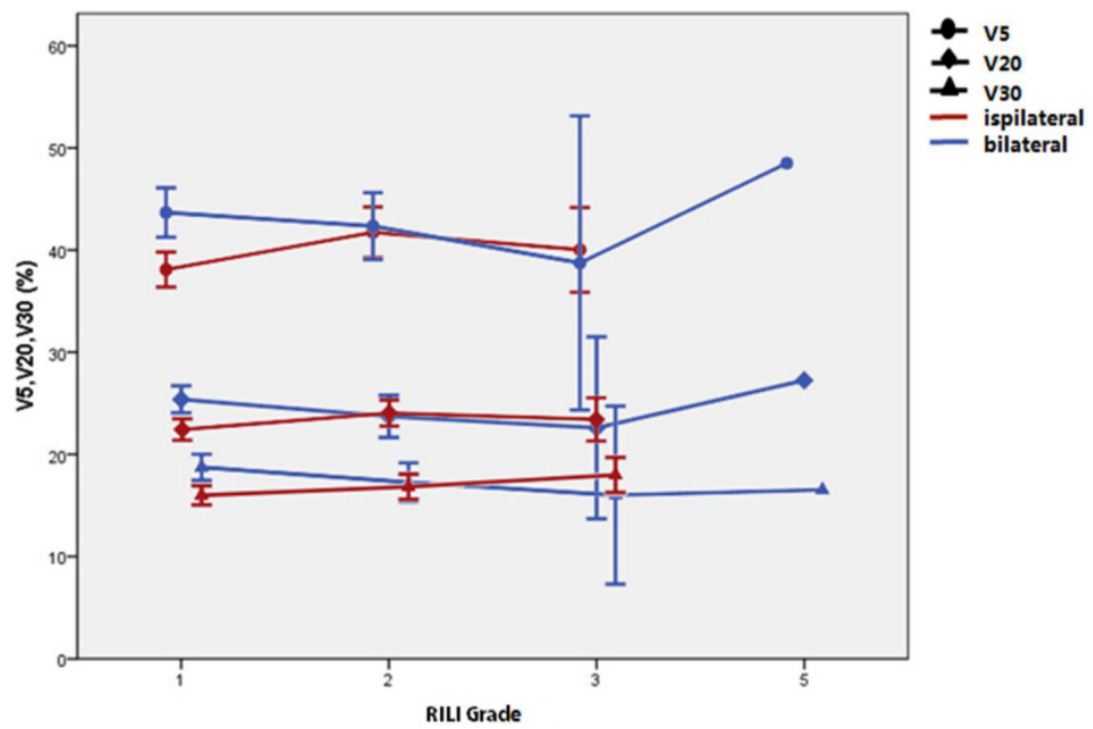

Figure 1. Mean and $95 \% \mathrm{Cl}$ of $\mathrm{V} 5, \mathrm{~V} 20$, and $\mathrm{V} 30$ for each grade of radiation-induced lung injury (RILI); cases of ipsilateral and bilateral injury are compared.

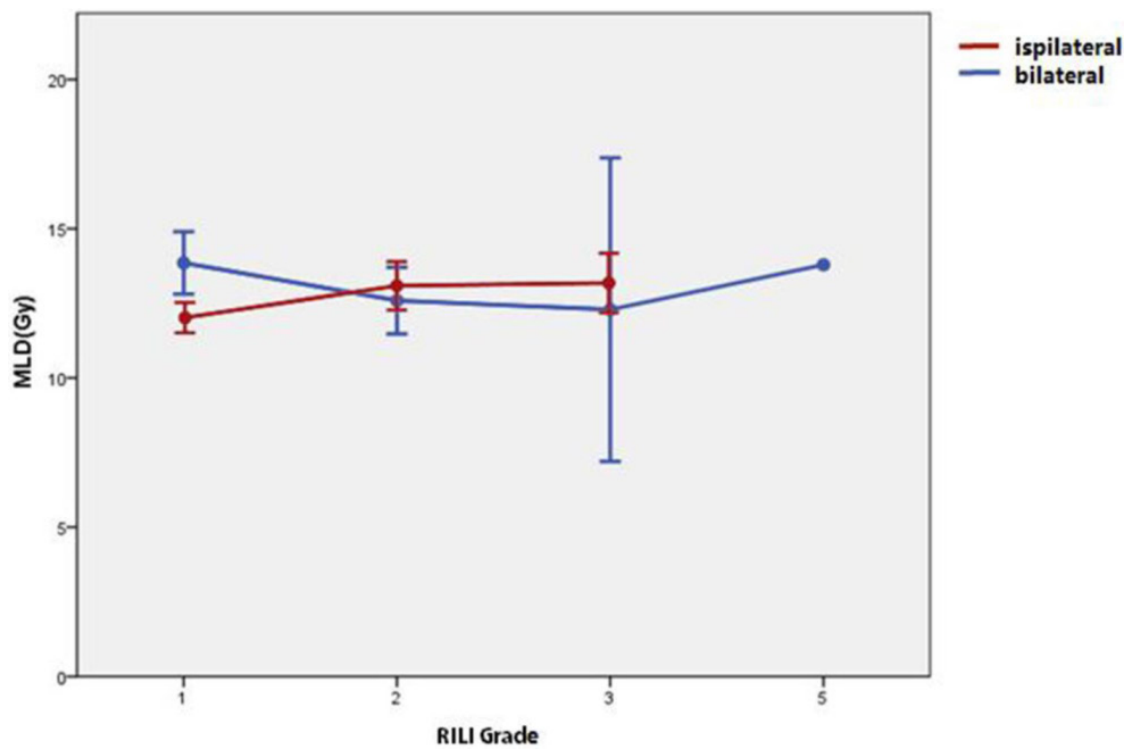

Figure 2. Mean and $95 \% \mathrm{Cl}$ of the mean lung dose (MLD) for each grade of radiation-induced lung injury (RILI); cases of ipsilateral and bilateral injury are compared. 
The results for radiation technology (IMRT vs. 3D-CRT) were found that the IMRT group had a higher incidence of RILI compared to 3D-CRT (38.2\% vs. $25.9 \%$ ), but this was not statistically significant. Vogelius et al ${ }^{19}$ proposed that there is interaction between chemotherapy and the radiation-dose distribution under clinical conditions. The effect of chemotherapy was modeled by the chemotherapy equivalent radiation dose (CERD). If CERD exceeded a certain threshold, for example $10 \mathrm{~Gy}^{19}$, the tomotherapy and IMRT plans were estimated to cause greater RILI than 3D-CRT. However, different results obtained from Sejpal et al ${ }^{20}$ and Shirvani et al ${ }^{21}$ seems not agree with Vogelius et al ${ }^{19}$. Regardless of the inconsistency of the above reports, the improved target conformity and higher dose delivered at specific sites makes IMRT more promising.

After univariate analysis, V5, V20, and MLD were found to be significantly associated with RILI, and to be highly correlated to each other with $r$ values from 0.744 to 0.861 . We choose MLD for multivariate analysis, and found that MLD was an independent risk factor for RILI. A number of studies have reported similar results showing that dosimetric parameters such as V5 9, V20 7,10-13, V30 8,12,14,15, MLD $4,7,12,15$ are strongly correlated to RILI. A consensus on Vdose and MLD thresholds does not currently exist, e.g., Wang et al found that a higher level of MLD (17.4 Gy-cutoff) identified by ROC analysis was significantly correlated with RILI 4, while MLD>16Gy and V30 $>18 \%$ was identified by ROC by Kim et al ${ }^{15}$. The optimized cut-off point determined by ROC is different when interpreting it clinically and statistically, thus Claude et al ${ }^{12}$ used thresholds based on the median Vdose rather than ROC, and found that $\mathrm{V} 20>18 \%, \mathrm{~V} 30>13 \%$, and $\mathrm{V} 40>10 \%$ were significantly associated with the incidence of RILI. In contrast to Roeder et al ${ }^{14}$ who divided the entire cohort into quartiles to achieve groups, Wang et al ${ }^{9}$ selected the cut-off point using martingale residuals between the two corresponding subgroups and identified V5 as the only independent factor by multivariate analysis (not V10-V65); 1-year actuarial incidences of grade $\geq 3$ pneumonitis in those with $\mathrm{V} 5 \leq 42 \%$ and those with V $5>42 \%$ were $3 \%$ and $38 \%$, respectively $(P=0.001)$. Our work confirmed the predictive value of dosimetric parameters and the need for more research into establishing a series of the optimized cut-off values for use in clinical practice.

In cases where the primary tumor was located unilaterally, we observed that RILI sometimes occurred bilaterally; this was found across all RILI grades, as shown in Table 3. The phenomenon is in part due to small amounts of radiation reaching the contralateral lung, though modern technology like
3D-CRT, IMRT, VMAT, and proton therapy enables more precise confinement of radiation to the target volume ${ }^{20,22}$. The bilateral lymphocytic alveolitis may also partly explain the phenomenon, as previous studies have revealed lymphocytosis in bilateral injury 23-25, and bilateral diffuse alveolar damage was observed histologically in 5 patients by Arbetter et al ${ }^{23}$ and in 2 patients by Fujita et al ${ }^{26}$. Hassaballa et al ${ }^{27}$ used PET to examine acute changes after radiotherapy in shielded lungs, and found that $81.2 \%(13 / 16)$ of patients showed increased FDG uptake in the shielded non-irradiated lung, and only one patient (6.2\%) developed clinically evident radiation pneumonitis, shown by diffuse, bilateral uptake of FDG. Our results show that the dosimetrics were only significantly increased in patients with grade- 1 bilateral RILI, but not in those with higher-grade injury. The distribution of grades between the bilateral and unilateral groups by a Mann-Whitney $U$ test indicated that more localized (ipsilateral) injury was associated with higher grade of injury, thus suggesting that additional contralateral lung injury may not correlate with a high risk of higher-grade RILI under the acceptable dosimetric parameter from DVH. On the base of the results, more attention should be paid on the contraint of dosimetric parameter values in radiation planning. It still deserves focus on whether extensive bilateral injury under higher dosimetric parameter values due to dose escalation correlates with higher-grade clinical manifestations.

It is worth noting a couple of limitations of this study. Since evaluation of symptomatic RILI was mainly dependent on medical records, bias in the patient selection could have influenced the analysis. The assessment of bilateral RILI was performed according to CT scans and the size of the lung damages was rudely neglected, which makes for a relatively crude result.

This retrospective study demonstrates a significant association between MLD and the risk of RILI. MLD was also strongly correlated to V5 and V20. Under the condition of acceptable conventional radiotherapy treatment planning, dosimetric parameters were higher in cases of bilateral RILI, but this increase was only significant for low-grade (non-symptomatic) RILI; furthermore, bilateral injury seems not be correlated to a high degree of RILI. To allow for greater dose escalation and improved treatment outcomes in the future, more research into minimizing radiation toxicity is clearly needed.

\section{Abbreviations}

RILI: radiation-induced lung injury; NSCLC: non-small-cell lung cancer; SCLC: small-cell lung cancer; CRT: chemoradiotherapy; KPS: Karnofsky 
performance status; IMRT: intensity-modulated radiotherapy; Vdose: the percentage lung volume irradiated to doses exceeding a threshold; MLD: mean lung dose; DVH: dose-volume histogram; RP: radiation pneumonitis; 3D-CRT: three-dimensional conformal radiotherapy; GTV: gross tumor volume; CTV: clinical target volume; PTV: planning target volume; CERD: chemotherapy equivalent radiation dose

\section{Acknowledgement}

This work was supported by grants from the Zhejiang Medicine \& Health Key Research Fund (No. 2012ZDA004) and the National Natural Science Foundation of China (No. 81272505/H1610).

Authors SH and FG contributed equally to this work.

\section{Competing Interests}

The authors have declared that no competing interest exists.

\section{References}

1. Kong FM, Ten Haken RK, Schipper MJ, et al. High-dose radiation improved local tumor control and overall survival in patients with inoperable/unresectable non-small-cell lung cancer: long-term results of a radiation dose escalation study. International journal of radiation oncology, biology, physics. 2005;63:324-333.

2. Warde P, Payne D. Does thoracic irradiation improve survival and local control in limited-stage small-cell carcinoma of the lung? A meta-analysis. Journal of clinical oncology : official journal of the American Society of Clinical Oncology. 1992;10:890-895.

3. Pignon JP, Arriagada R, Ihde DC, et al. A meta-analysis of thoracic radiotherapy for small-cell lung cancer. The New England journal of medicine. 1992;327:1618-1624.

4. Wang J, Cao J, Yuan S, et al. Poor baseline pulmonary function may not increase the risk of radiation-induced lung toxicity. International journal of radiation oncology, biology, physics. 2013;85:798-804

5. McCurdy M, Bergsma DP, Hyun E, et al. The Role of Lung Lobes in Radiation Pneumonitis and Radiation-Induced Inflammation in the Lung: A Retrospective Study. Journal of radiation oncology. 2013;2:203-208.

6. Leprieur EG, Fernandez D, Chatellier G, Klotz S, Giraud P, Durdux C. Acute radiation pneumonitis after conformational radiotherapy for nonsmall cell lung cancer: clinical, dosimetric, and associated-treatment risk factors. Journal of cancer research and therapeutics. 2013;9:447-451.

7. Zhang J, Li B, Ding X, et al. Genetic variants in inducible nitric oxide synthase gene are associated with the risk of radiation-induced lung injury in lung cancer patients receiving definitive thoracic radiation. Radiotherapy and oncology : journal of the European Society for Therapeutic Radiology and Oncology. 2014:111:194-198.

8. Fay M, Tan A, Fisher R, Mac Manus M, Wirth A, Ball D. Dose-volume histogram analysis as predictor of radiation pneumonitis in primary lung cancer patients treated with radiotherapy. International journal of radiation oncology, biology, physics. 2005;61:1355-1363.

9. Wang S, Liao Z, Wei X, et al. Analysis of clinical and dosimetric factors associated with treatment-related pneumonitis (TRP) in patients with non-small-cell lung cancer (NSCLC) treated with concurrent chemotherapy and three-dimensional conformal radiotherapy (3D-CRT). International journal of radiation oncology, biology, physics. 2006;66:1399-1407.

10. Graham MV, Purdy JA, Emami B, et al. Clinical dose-volume histogram analysis for pneumonitis after 3D treatment for non-small cell lung cancer (NSCLC). International journal of radiation oncology, biology, physics. 1999;45:323-329.

11. Tsujino K, Hirota S, Endo M, et al. Predictive value of dose-volume histogram parameters for predicting radiation pneumonitis after concurrent chemoradiation for lung cancer. International journal of radiation oncology, biology, physics. 2003;55:110-115.

12. Claude L, Perol D, Ginestet C, et al. A prospective study on radiation pneumonitis following conformal radiation therapy in non-small-cell lung cancer: clinical and dosimetric factors analysis. Radiotherapy and oncology : journal of the European Society for Therapeutic Radiology and Oncology. 2004;71:175-181.

13. Salama JK, Stinchcombe TE, Gu L, et al. Pulmonary toxicity in Stage III non-small cell lung cancer patients treated with high-dose (74 Gy) 3-dimensional conformal thoracic radiotherapy and concurrent chemotherapy following induction chemotherapy: a secondary analysis of Cancer and Leukemia Group B (CALGB) trial 30105. International journal of radiation oncology, biology, physics. 2011;81:e269-274.

14. Roeder F, Friedrich J, Timke C, et al. Correlation of patient-related factors and dose-volume histogram parameters with the onset of radiation pneumonitis in patients with small cell lung cancer. Strahlenther Onkol. 2010;186:149-156.

15. Kim M, Lee J, Ha B, Lee R, Lee KJ, Suh HS. Factors predicting radiation pneumonitis in locally advanced non-small cell lung cancer. Radiation oncology journal. 2011;29:181-190.

16. Yamada M, Kudoh S, Hirata K, Nakajima T, Yoshikawa J. Risk factors of pneumonitis following chemoradiotherapy for lung cancer. European journal of cancer. 1998;34:71-75.

17. Rancati T, Ceresoli GL, Gagliardi G, Schipani S, Cattaneo GM. Factors predicting radiation pneumonitis in lung cancer patients: a retrospective study. Radiotherapy and oncology : journal of the European Society for Therapeutic Radiology and Oncology. 2003;67:275-283.

18. Robnett TJ, Machtay M, Vines EF, McKenna MG, Algazy KM, McKenna WG. Factors predicting severe radiation pneumonitis in patients receiving definitive chemoradiation for lung cancer. International journal of radiation oncology, biology, physics. 2000;48:89-94

19. Vogelius IS, Westerly DC, Cannon GM, et al. Intensity-modulated radiotherapy might increase pneumonitis risk relative to three-dimensional conformal radiotherapy in patients receiving combined chemotherapy and radiotherapy: a modeling study of dose dumping. International journal of radiation oncology, biology, physics. 2011;80:893-899.

20. Sejpal S, Komaki R, Tsao A, et al. Early findings on toxicity of proton beam therapy with concurrent chemotherapy for nonsmall cell lung cancer. Cancer. 2011;117:3004-3013.

21. Shirvani SM, Jiang J, Gomez DR, Chang JY, Buchholz TA, Smith BD. Intensity modulated radiotherapy for stage III non-small cell lung cancer in the United States: predictors of use and association with toxicities. Lung cancer. 2013;82:252-259.

22. Bertelsen A, Hansen O, Brink C. Does VMAT for treatment of NSCLC patients increase the risk of pneumonitis compared to IMRT ? - a planning study. Acta oncologica. 2012;51:752-758.

23. Arbetter KR, Prakash UB, Tazelaar HD, Douglas WW. Radiation-induced pneumonitis in the "nonirradiated" lung. Mayo Clinic proceedings. 1999;74:27-36.

24. Andreo Garcia F, Garro Martinez P, Torrabadella de Reynoso P, Ojanguren Saban I, Esquirol Puig X, Mesalles Sanjuan E. Bilateral pneumonia after localized irradiation of a thymoma. Description of a case. Archivos de bronconeumologia. 1996;32:544-546.

25. Roberts CM, Foulcher E, Zaunders JJ, et al. Radiation pneumonitis: a possible lymphocyte-mediated hypersensitivity reaction. Annals of internal medicine. 1993;118:696-700.

26. Fujita J, Bandoh S, Ohtsuki Y, et al. The role of anti-epithelial cell antibodies in the pathogenesis of bilateral radiation pneumonitis caused by unilateral thoracic irradiation. Respir Med. 2000;94:875-880.

27. Hassaballa HA, Cohen ES, Khan AJ, Ali A, Bonomi P, Rubin DB. Positron emission tomography demonstrates radiation-induced changes to nonirradiated lungs in lung cancer patients treated with radiation and chemotherapy. Chest. 2005;128:1448-1452. 